\title{
Distinct Effects of Social Stress on Working Memory in Obsessive-Compulsive Disorder
}

\author{
Qianqian $\mathrm{Li}^{1,2} \cdot$ Jun Yan ${ }^{1,2} \cdot J^{1, n m i n}$ Liao $^{1,2} \cdot$ Xiao Zhang $^{1,2}$ (D) $\cdot$ Lijun Liu ${ }^{1,2} \cdot$ \\ Xiaoyu Fu ${ }^{1,2} \cdot$ Hao Yang $\operatorname{Tan}^{5,6} \cdot$ Dai Zhang ${ }^{1,2,3,4}$ (1) Hao Yan $^{1,2}$ (1)
}

Received: 12 December 2019/Accepted: 13 May 2020/Published online: 1 October 2020

(C) The Author(s) 2020

\begin{abstract}
Stress might exaggerate the compulsion and impair the working memory of patients with obsessivecompulsive disorder (OCD). This study evaluated the effect of stress on the cognitive neural processing of working memory in OCD and its clinical significance using a "number calculation working memory" task. Thirty-eight patients and 55 gender- and education-matched healthy controls were examined. Stress impaired the performance of the manipulation task in patients. Healthy controls showed less engagement of the medial prefrontal cortex
\end{abstract}

Qianqian Li and Jun Yan have contributed to this work.

Electronic supplementary material The online version of this article (https://doi.org/10.1007/s12264-020-00579-3) contains supplementary material, which is available to authorized users.

Dai Zhang

daizhang@bjmu.edu.cn

$\bowtie$ Hao Yan

hao_y@bjmu.edu.cn

1 Peking University Sixth Hospital, Beijing 100191, China

2 Peking University Institute of Mental Health, National Health Commission Key Laboratory of Mental Health (Peking University), National Clinical Research Center for Mental Disorders (Peking University Sixth Hospital), Beijing 100191, China

3 Tsinghua University-Peking University Joint Center for Life Sciences, Beijing 100871, China

4 PKU-IDG/McGovern Institute for Brain Research, Peking University, Beijing 100871, China

5 Lieber Institute for Brain Development, Baltimore, MD 21205, USA

6 Department of Psychiatry and Behavioral Sciences, Johns Hopkins University School of Medicine, Baltimore, MD 21205, USA and striatum during the task under stress versus less stress, which was absent in the patients with OCD. The diagnosis $\times$ stress interaction effect was significant in the right fusiform, supplementary motor area, precentral cortex and caudate. The failure of suppression of the medial prefrontal cortex and striatum and stress-related hyperactivation in the right fusiform, supplementary motor area, precentral cortex, and caudate might be an OCD-related psychopathological and neural response to stress.

Keywords Working memory · Acute stress - Obsessivecompulsive disorder - Functional magnetic resonance imaging

\section{Introduction}

Obsessive-compulsive disorder (OCD) is a chronic and disabling mental disease [1, 2] characterized by the presence of obsessions (repeated thoughts) or compulsions (repeated behaviors to neutralize anxiety), or both [3]. The World Health Organization ranks it as one of the ten most disabling conditions by lost income and reduced quality of life [4].

Stress is one of the main risk factors for the formation of the disorder [5], and previous studies have indicated that OCD patients have an impaired stress response [6]. The neuropsychological theories of OCD suggest that it might be a disorder of imbalance between goal-directed behavior and maladaptive habits [7]. The excessive habit formation or habit dysregulation might result in the compulsions in OCD, which can be exaggerated by stress [8]. Moreover, stress impairs working memory (WM) and cognitive flexibility; this has been confirmed by meta-analysis of the main neuropsychological assessments in OCD patients [9]. 
Meanwhile, WM has been found to shape and moderate the balance between the goal-directed and habitual systems as one of the core cognitive abilities [10, 11].

Accumulating evidence from neuroimaging studies has suggested a certain extent of spatial overlap between the neural mechanism of OCD, the stress effect, and the WM process [12]. The well-known neuropathological model suggests that the function of the cortical-striatal-thalamocortical (CSTC) loop is impaired in OCD [13-15]. Banca et al. found that, during effective symptom provocation, OCD patients show reduced activation in the ventromedial prefrontal cortex (vmPFC) and caudate nucleus, and increased activation in regions of the pre-supplementary motor area (pre-SMA) and putamen, which have also been implicated in goal-directed behavioral control and habit learning, respectively [16]. A visuospatial WM study suggested an inefficient fronto-parietal executive network and increased connectivity between frontal regions and the amygdala in OCD [17]. Moreover, meta-analysis of the brain responses to the stress of trauma revealed hyperactivation in the amygdala, insula, and precuneus cortex and hypoactivation in the anterior cingulate cortices and vmPFC $[18,19]$. Some studies have indicated that stress leads to changes in the neuroendocrine and limbic brain, resulting in mental diseases such as depression and posttraumatic stress disorder [20, 21]. However, how stress influences the neural processing related to the cognitive deficits of WM in OCD and the clinical correlations remain unknown.

Therefore, in the current study, a new event-related "number calculation working memory" task which integrated interpersonally competitive stress into the WM process was used to investigate the stress effect on WM in OCD. This newly designed task differs from the Montreal Imaging Stress Task (MIST) [22] which consists of mental arithmetic under social evaluating stress but not WM. Based on our previous work in a large sample of healthy adult Han Chinese individuals [23], the task-related activity included the prefrontal-parietal system and stress-related suppression of the medial prefrontal cortex (mPFC) and striatum. Therefore, in view of the neuropathological theory of OCD, we hypothesized that the regions of the CSTC circuit, frontal-parietal executive, and stress-related networks might respond differentially to the WM task under stress in patients with OCD versus healthy controls.

\section{Materials and Methods}

\section{Ethics Statement}

The Ethics Committee of the Peking University Sixth Hospital approved the study. All participants were given detailed information regarding the purpose and procedures of the study. Only patients who could consent were invited to participate. All the participants enrolled gave written consent.

\section{Participants}

In total, 125 individuals participated in the study: 58 patients with OCD and 67 healthy controls (HCs). The patients were recruited from either the inpatient or outpatient department of Peking University Sixth Hospital. Patients all met the Diagnostic and Statistical Manual of Mental Disorders, 4th Edition, Text Revision (DSM-IVTR) diagnostic criteria of OCD, without other comorbidities in the DSM-IV-TR Axis I Disorders (including depression), and were evaluated by two psychiatrists using the Structured Clinical Interview for DSM-IV-TR Axis I Disorders, Patient Edition (SCID-I/P). The exclusion criteria were any comorbidity of other SCID-I diagnoses, electroconvulsive therapy within six months, or a history of severe medical illness. All HCs were recruited from the local community by advertisement and assessed by psychiatrists using the SCID-I, Non-Patient Edition to exclude any mental disorder. Participants were excluded if they had: a history of neurological disease, a history of $>5 \mathrm{~min}$ loss of consciousness, or magnetic resonance imaging (MRI) contraindications.

The Yale-Brown Obsessive Compulsive Symptom Checklist Scale and Severity Scale (YBOCS-CS/YBOCSSS) [24], Hamilton Anxiety Scale (HAMA) and Hamilton Depression Scale (HAMD), and four factors were used to assess the symptom severity and dimensions in patients. In the 38 patients included in the final analysis, 24 were taking one or more antidepressants and 14 were drug-naïve. All medication doses were transformed into Imipramine equivalents (mg/day) [25] (see Tables 1 and $\mathrm{S} 1$ for details).

\section{Working Memory Paradigm}

We applied a new event-related "number calculation working memory" task from previous work, which comprised alternating competitive and non-competitive blocks (NumComp-task) [23] (Fig. 1). The participants received task training before MRI scanning to ensure that they understood the task well.

During the trials with competition (C), participants were led to believe that they were playing a number calculation game against a competitor of similar age and gender, and were judged based on timing and accuracy. The NumComp-task had two sessions: 14 competitive trials and 14 non-competitive trials in total. To induce social stress, participants received more negative feedback of "you lost" (5/7) among the 14 competitive trials. During the non- 
Table 1 Demographics and clinical data of the obsessivecompulsive disorder patients (OCD) and healthy controls (HC).

\begin{tabular}{lllrl}
\hline Variables & OCD $(n=38)^{*}$ & HC $(n=55)^{*}$ & $t / \chi^{2}$ & $P$ value \\
\hline Gender (F/M) & $14 / 24$ & $30 / 25$ & 2.83 & $0.09^{* *}$ \\
Age (years) & $27.1 \pm 6.1$ & $23.6 \pm 2.9$ & 3.25 & $0.02 \dagger$ \\
Edu (years) & $15.7 \pm 2.7$ & $16.4 \pm 1.6$ & -1.32 & $0.19 \dagger$ \\
Imipramine equivalents (mg/day) & $232.6 \pm 128.8$ & & \\
Course (months) & $77.0 \pm 47.1$ & & \\
YBOSC-TS & $21.9 \pm 13.8$ & & \\
YBOSS-TS & $23.0 \pm 7.8$ & & \\
HAMAS & $12.0 \pm 7.6$ & & & \\
HAMDS & $8.9 \pm 6.2$ & & & \\
\hline
\end{tabular}

*Unless otherwise indicated, data are the mean $\pm \mathrm{SD} ; * *$ Pearson $\chi^{2}$ (categorical data); †independentsample $t$-test (parametric data); YBOSC-TS, Yale-Brown Symptom Checklist total score; YBOSS-TS, Yale-Brown severity scale total score; HAMAS, Hamilton Anxiety Scale total score; HAMDS, Hamilton Depression Scale total score.

\section{Competitive Block}

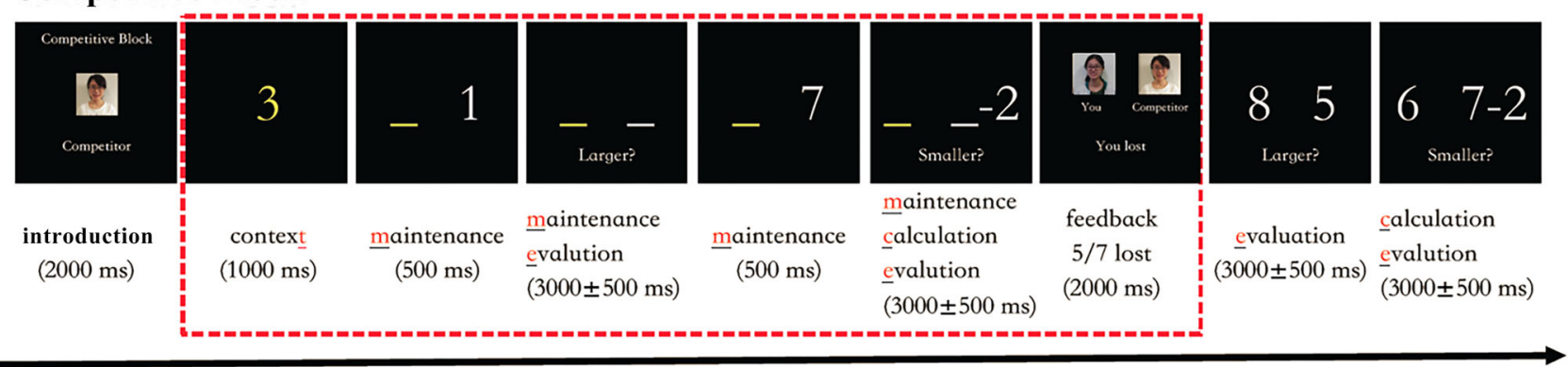

\section{Non-competitive Block}

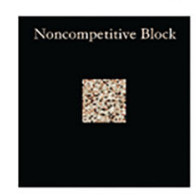

introduction (2000 ms)

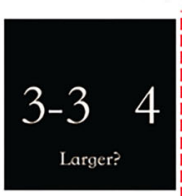

calculation evalution $(3000 \pm 500 \mathrm{~ms})$

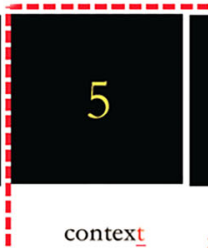

context
$(1000 \mathrm{~ms})$
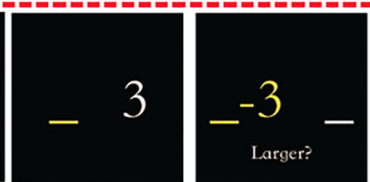

maintenance

maintenance calculation

(500 ms)

evalution $(3000 \pm 500 \mathrm{~ms})$

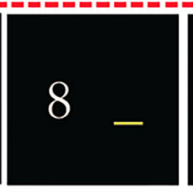

maintenance

(500 ms)

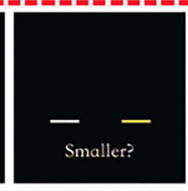

maintenance

evalution

$(3000 \pm 500 \mathrm{~ms})$

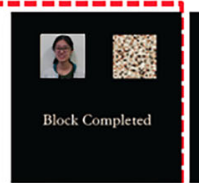

43

Smaller?

feedback evaluation

$(2000 \mathrm{~ms}) \quad$ i $(3000 \pm 500 \mathrm{~ms})$

Fig. 1 The event-related numerical WM task paradigm.

competitive (NC) trials, the participants played the game with no competitor and received a neutral response.

For each trial under the WM conditions, the participants were presented with two numbers from 1 to 9 successively to encode into WM and subsequently had to be maintained. Then they were asked which of the two results was "larger" or "smaller" as instructed (maintenance evaluation, $\mathrm{ME}$ ) or had to perform mental arithmetic (subtraction) on one of the numbers before giving a response to the "larger" or "smaller" comparison (maintenance calculation evaluation, MCE). Further details of the NumComp-task are available in the supplementary material.
The NumComp-task had competitive and non-competitive blocks. For WM (enclosed in red dashes), participants encoded 2 integer numbers in yellow or white presented over $1 \mathrm{~s}$ and to be retained in WM (underlined in the same color, yellow or white) across an interval of 3-5 s. In maintenance trials, participants responded to which of the two numbers was "larger" or "smaller" within 2.5-3.5 s. In the manipulation trials, participants had to do mental arithmetic on one of the two numbers in yellow or white before the "larger" or "smaller" evaluation within 2.5-3.5 s. These two kinds of trials were embedded within equal numbers of competitive or non-competitive blocks. Two blocks were counterbalanced in 2 runs. Each run 
took $\sim 10 \mathrm{~min}$. All the instructions were originally in Chinese. All "competitors" were of the same gender and age. The interspersed fixation was between each complete competitive block and a noncompetitive block and was not shown.

\section{Image Acquisition}

Imaging data were acquired using a 3.0-Tesla GE scanner (Discovery MR750) at the Center for Neuroimaging, Peking University Sixth Hospital. Foam pads were used to minimize head motion. A gradient echo sequence was used to acquire blood oxygen level-dependent (BOLD) functional MRI (fMRI) images, and each volume consisted of 33 axial slices covering the entire cerebrum and cerebellum with the following parameters: thickness $/$ gap $=4.2 \mathrm{~mm} / 0 \mathrm{~mm}, \quad$ TR/TE $=2000 / 30 \mathrm{~ms}, \quad$ flip angle $=90^{\circ}$, field of view $=22.4 \mathrm{~cm} \times 22.4 \mathrm{~cm}$, matrix $=$ $64 \times 64$. Three dummy scans were acquired at the beginning of the fMRI scanning.

\section{Image Processing and Analyses}

We excluded participants with an accuracy rate $<50 \%$ in any WM conditions ( 8 OCDs), those with head motion $>$ $3 \mathrm{~mm}$ translation or $3^{\circ}$ rotation ( 8 OCDs and $6 \mathrm{HCs}$ ), and those with image artifacts or did not complete the task (4 OCDs and $6 \mathrm{HCs}$ ) for quality control. In total, 93 participants (38 OCDs and $55 \mathrm{HCs}$ ) were included in the final analysis (Table 1).

The fMRI imaging data were pre-processed using MatLab 2016b and SPM12 (http://www.fil.ion.ucl.ac.uk/ spm). Functional images for each participant was slicetiming corrected, realigned to the first volume in the time series, and corrected for head motion. Images were then spatially normalized into standard stereotaxic space (Montreal Neurological Institute template) using fourthdegree B-spline interpolation. Spatial smoothing was applied with a Gaussian filter set at $8 \mathrm{~mm}$ full-width at half-maximum. Each task-evoked stimulus was modelled as a separate delta function and convolved with a canonical hemodynamic response function; ratio normalized to the whole-brain global mean to control for systematic differences in global activity, and temporally filtered using a high-pass filter of $128 \mathrm{~s}$. Each task-evoked stimulus event was modelled for correctly-performed trials in the general linear model of the first-level analysis of the image data. Incorrect responses and residual movement parameters were also modelled as regressors of no interest [26].

The planned contrasts of interest for a second-level random effect within-group analysis using one-sample t-tests were brain activity in the maintenance (ME) or manipulation (MCE) task conditions under non-competitive (NC) and competitive (C) condition at $P<0.05$, whole-brain family-wise error (FWE) corrected.

For the diagnosis effect (group difference) on brain activity in each WM condition (ME or MCE) under competitive or non-competitive condition, the significance level was set as an uncorrected $P<0.001$, with a cluster sizes $\geq 86$ voxels $\left(688 \mathrm{~mm}^{3}\right)$ for $\mathrm{ME} \_\mathrm{C}, \geq 94$ voxels $\left(752 \mathrm{~mm}^{3}\right)$ for $\mathrm{ME} \_\mathrm{NC}, \geq 83$ voxels $\left(664 \mathrm{~mm}^{3}\right)$ for MCE_C, and $\geq 103$ voxels $\left(824 \mathrm{~mm}^{3}\right)$ for MCE_NC with independent-sample $t$-tests, which corresponded to a corrected $P<0.05$ determined by 5000 Monte Carlo simulations using AlphaSim correction within the grey matter mask in DPABI_V4.0 (http://rfmri.org/dpabi). The intersubject variability was treated as a random effect, controlled for age, gender, and years of education.

For the stress effect indicated as brain activity during the maintenance or manipulation task conditions under competition versus non-competition in the HC and OCD groups, the significance level was set as an uncorrected $P<0.001$, with cluster sizes $\geq 125 / 124$ voxels $\left(1000 / 992 \mathrm{~mm}^{3}\right)$ for $\mathrm{HC}$ and $\geq 137 / 229$ voxels $\left(1096 / 1832 \mathrm{~mm}^{3}\right)$ for OCD with onesample $t$-tests, which corresponded to a corrected $P<0.05$ determined by 5000 Monte Carlo simulations using AlphaSim correction within the grey matter mask in DPABI_V4.0 (http://rfmri.org/dpabi). The imipramine equivalent was included as a covariate to control for the medication effect in the OCD within-group analysis.

The contrast images of the competitive or non-competitive condition were subsequently subjected to a flexible $2 \times 2$ analysis of variance (ANOVA) to explore the brain activity of the diagnosis $\times$ stress interaction effect separately in the $\mathrm{ME}$ and $\mathrm{MCE}$ conditions. The significance level was set at an uncorrected $P<0.001$, with cluster sizes $\geq 38$ voxels $\left(304 \mathrm{~mm}^{3}\right)$ for maintenance and $\geq 95$ voxels $\left(760 \mathrm{~mm}^{3}\right)$ for manipulation, which corresponded to a corrected $P<0.05$ determined by 5000 Monte Carlo simulations using AlphaSim correction within the grey matter mask in DPABI_V4.0 (http://rfmri.org/dpabi). Around the peak coordinates, an $8 \mathrm{~mm}$ radius sphere was created using DPABI_V4.0 as the region of interest (ROI) of each area showing a significant interaction effect, and then the contrast values of the ROIs from the corresponding contrast images of each task and condition in each group were extracted for a two-way repeated measures ANOVA and a simple main effect analysis using GraphPad Prism 7.0 (https://www.graphpad.com). The significance level of the ANOVA and simple main effect analysis in GraphPad Prism 7.0 was set at $P<0.05$, false discovery rate (FDR) correction. 


\section{Statistical Analyses of Clinical and Behavioral Data}

Demographic and clinical data were analyzed with a standard statistical package (IBM SPSS 21.0, Chicago, IL), using the $t$-test and $\chi^{2}$ test. Task behavioral data (accuracy rate and reaction time) of the two groups under stress and non-stress condition were analyzed with GraphPad Prism 7.0 using two-way repeated measures ANOVA. If the diagnosis $\times$ stress interaction effect was significant, a simple main effect analysis was applied as a post hoc analysis in GraphPad Prism 7.0. If the brain activity of diagnosis $\times$ stress interaction effect was significant in ME or MCE conditions, the stress-related contrast values of the ROI of the interaction effect from the corresponding contrast images of each task condition (ME or MCE under competitive versus non-competitive contrast images) in the OCD group were extracted and correlated to the clinical variables (Y-BOCS, HAMA, and HAMD) and task behavioral performance. The significance level of the $t$-test and $\chi^{2}$ test was set at $P<0.05$. The significance level of the two-way repeated measures ANOVA, simple main effect, and correlation analysis was set at $P<0.05$, with FDR correction.

\section{Results}

\section{Behavioral Performance}

In terms of the accuracy rate of maintenance, we only found significant main effects of stress (competitive $v s$ non-competitive, $F=38.47, P<0.0001$ ). The accuracy was higher during the competitive than the non-competitive condition in OCDs $(t=3.48, q=0.0008$, FDR correction) and HCs $(t=5.52, q<0.0001$, FDR correction) (Fig. 2A and Table S2). As for the accuracy rate in the manipulation task, the main effect of diagnosis (OCD vs HC, $F=4.89, P=0.03$ ) and the interaction effects of diagnosis $\times$ stress $(F=7.47, P=0.008)$ were significant. The accuracy was lower in OCDs than in HCs only during the competitive condition $(t=3.32, q=0.001$, FDR correction) and the accuracy was lower under the competitive than the non-competitive condition only in OCDs $(t=2.57, \quad q=0.01$, FDR correction) (Fig. 2C and Table S2-S3).

In terms of reaction time in the maintenance and manipulation tasks, the main effects of diagnosis $(F=4.87, P=0.03, \mathrm{ME} ; F=8.54, P=0.004, \mathrm{MCE})$ and stress $(F=5.02, P=0.03$, only in ME) were significant and there was no significant diagnosis $\times$ stress interaction effect (Table S2). The reaction time was longer in OCDs than that in HCs during both the competitive $(t=2.20, q=0.043$, FDR correction, ME; $t=3.31$, $q=0.002$, FDR correction, MCE) and non-competitive $(t=2.06, q=0.043$, FDR correction, ME; $t=2.27$, $q=0.03$, FDR correction, MCE) conditions. The reaction time was shorter under the competitive than the noncompetitive ME condition. However, there was no significant stress effect within each group (Fig. 2B and Fig. 2D).

\section{Brain Activation}

\section{WM-Related Brain Activation}

During each of the WM maintenance and manipulation tasks under the competitive or non-competitive condition in both OCDs and HCs, regions in the prefrontal, parietal, temporal, occipital, and cerebellar cortices, and the striatum were robustly activated, along with well-established deactivation in areas of the default mode network (DMN) during the cognitive task, including the mPFC and posterior cingulate cortex $(P<0.05$, whole-brain FWE correction for multiple comparisons, see Figs. 3 and 4, left).

There was no significant between-group difference in the brain activity in WM maintenance or manipulation under competitive and non-competitive conditions.

\section{Stress Effect on Brain Activity in Each Group}

In terms of stress effects on WM maintenance and manipulation, a pattern of less engagement of the basal ganglia (less activation) and mPFC (more deactivation), more activation of the cerebellum was found during maintenance and manipulation under competitive versus non-competitive conditions in HCs $(P<0.05$, AlphaSim correction for multiple comparisons, see Fig. 3, right, and Table S4).

The pattern of reduced engagement of the basal ganglia and $\mathrm{mPFC}$ in HCs was absent from the OCDs. Taking the WM manipulation for example, the more activated regions included the dorsal and ventral anterior cingulate, superior temporal lobe, bilateral hippocampus, bilateral thalamus, and bilateral SMA which are implicated in the neural processing of goal-directed behavioral control, habit learning, and stress; while the less deactivated regions included the medial frontal gyrus and bilateral temporal cortex, which are implicated in the DMN $(P<0.05$, AlphaSim correction for multiple comparisons, see Fig. 4, right, and Table S5).

\section{Diagnosis and Stress Interaction Effects}

For the WM manipulation task, we found a significant diagnosis $\times$ stress interaction effect in the right fusiform cortex, right supplementary motor area (SMA), right 


\section{A Two-way ANOVA}

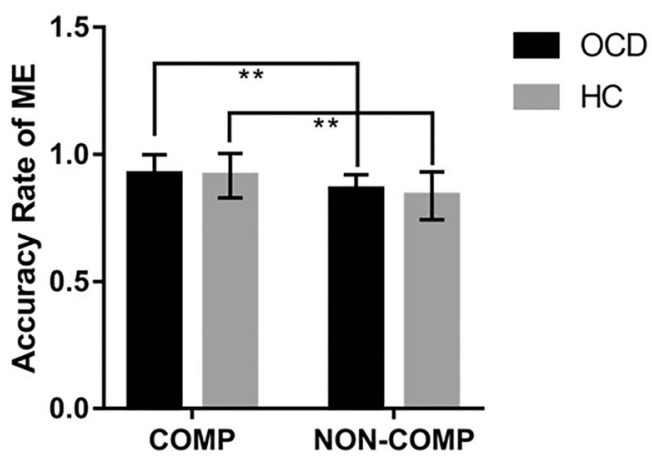

C

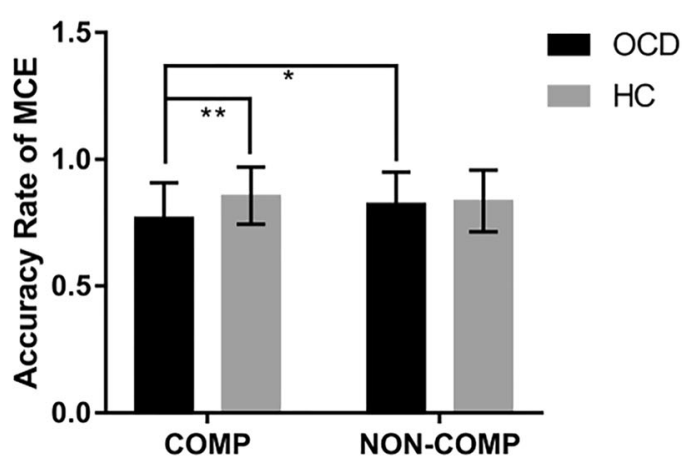

Fig. 2 Results of two-way ANOVA of diagnosis and stress and multiple comparisons in behavioral performance. A Stress has the main effect on accuracy rate in the ME task. The accuracy rate is higher under competition than non-competition in both groups. B, D Diagnosis has the main effect on average reaction time in the ME and MCE tasks. The average reaction time is longer in OCDs than HCs under both competitive and non-competitive conditions. C Stress

precentral cortex, and right caudate $(P<0.05$, AlphaSim correction for multiple comparisons, Fig. 5A and Table 2). The contrast values of the four ROIs in WM manipulation under competitive or non-competitive conditions of each group were extracted for further analysis. The activation of all four areas was significantly higher under competitive than non-competitive conditions only in OCDs (fusiform, $t=4.66, q<0.0001$; SMA, $t=4.63, q<0.0001$; precentral cortex, $t=4.37, q<0.0001$; caudate, $t=2.83$, $q=0.01$; FDR correction; Fig. 5B-E and Tables S6$\mathrm{S} 10)$. The activation of the right caudate was lower under competitive versus non-competitive conditions only in HCs $(t=2.53, \quad q=0.01, \quad$ FDR correction; Fig. 5E and Table S10). In conclusion, the stress effect on brain activity differed between OCDs and HCs. The right fusiform, SMA, precentral cortex, and caudate had increased stress-related activity in OCDs, but not in $\mathrm{HCs}$, while the right caudate had decreased stress-related activity in HCs.
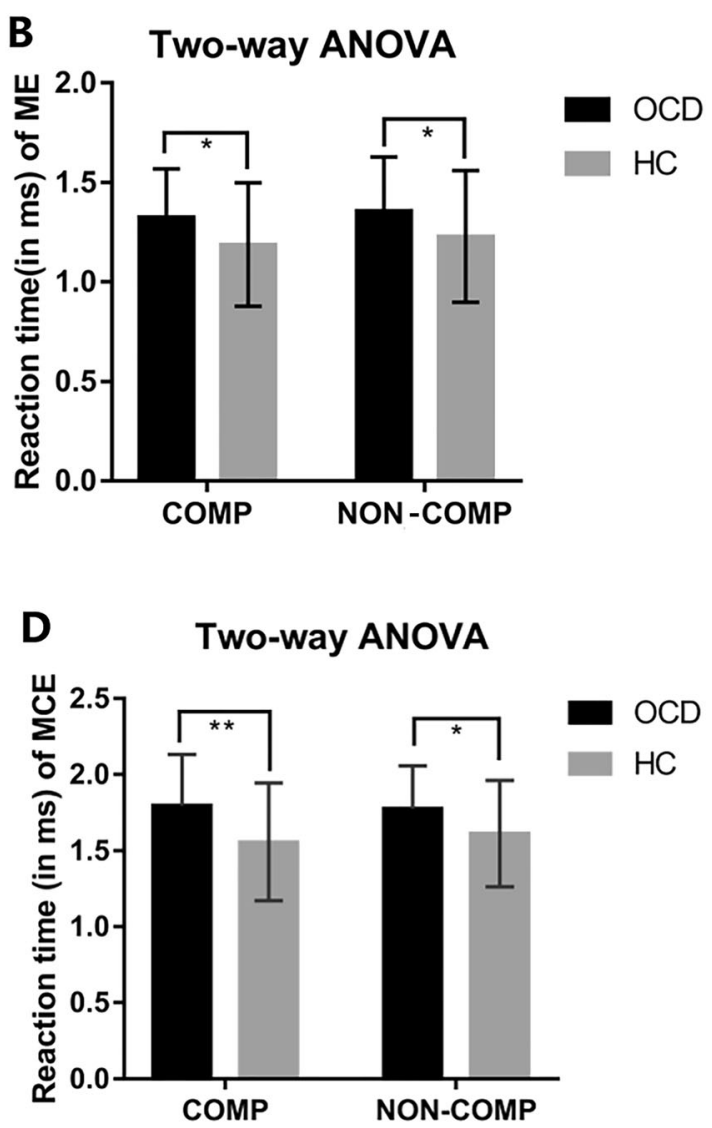

and diagnosis have an interaction effect on accuracy rate in the MCE task. The accuracy rate is lower in OCDs than HCs only under the competitive condition and the accuracy rate is lower under competitive than non-competitive conditions only in OCDs. ME, maintenance evaluation; $\mathrm{MCE}$, maintenance calculation evaluation; $\mathrm{C}$, competition; NC, non-competition $* P<0.05$, $* * P<0.01$.

No significant brain activity of diagnosis $\times$ stress interaction effect was found for WM maintenance.

\section{Correlation Analysis}

Correlation analyses were carried out between the stressrelated activity of the brain regions with a significant diagnosis $\times$ stress interaction and the stress-related behavioral measures in OCDs. Considering the stress effect was only significant in accuracy rate but not in reaction time in OCDs (Fig. 2), the measure of the task performance only included the accuracy rate difference between manipulation under competitive and non-competitive condition.

In the OCD patients, the severity of obsessions and compulsions was positively correlated with their anxiety and depression symptoms (Table S11). Behavioral performance of the maintenance and manipulation tasks was significantly correlated with symptom severity (worse symptoms of depression correlated with faster responses), 


\section{$\mathrm{HC}$}

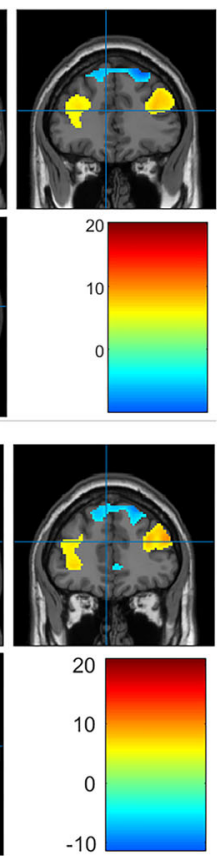

COMP
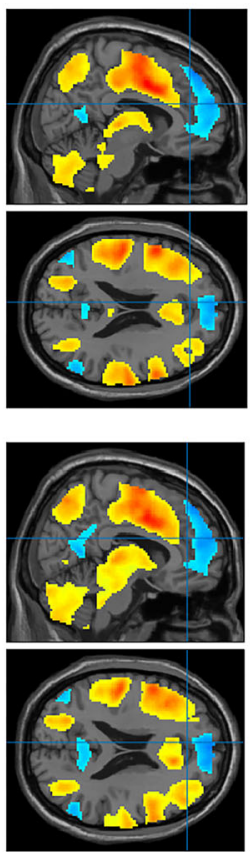

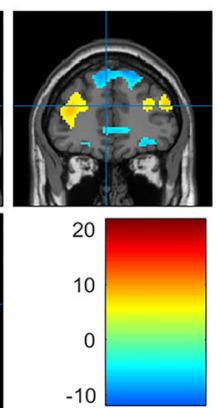

$-10$
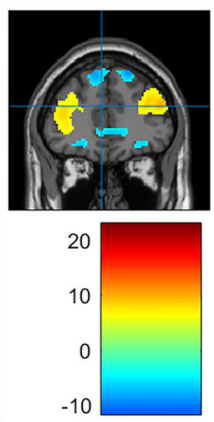

COMP vs. NON-COMP
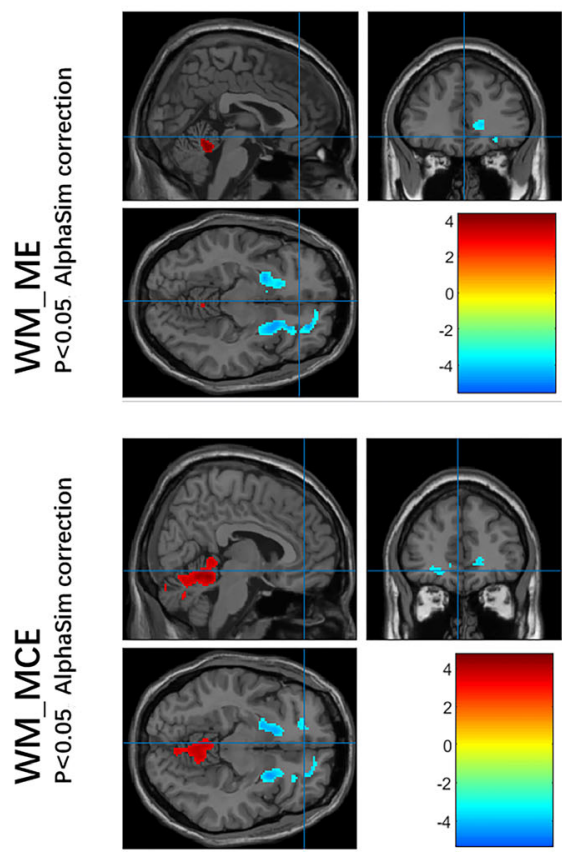

correction for multiple comparisons). ME, maintenance evaluation; MCE, maintenance calculation evaluation; COMP, competition; NON-COMP, non-competition; FWE, whole-brain family-wise error corrected. voxels). B Activity of competitive versus non-competitive conditions group under different task patterns (right, $P<0.05$, AlphaSim

and more severe obsession symptoms were associated with a lower accuracy rate: obsession severity factors were negatively correlated with the accuracy rate in the manipulation task under stress $(r=-0.479, P<0.01 ; r=-$ $0.358, P<0.05)$ (Table S12).

In terms of the correlation between the neural activation of the stress effect with the symptoms and task performance, only significantly negative correlations were found with the clinical symptom severity (Table S13), such as a stress-related activation change in the right fusiform and SMA showing negative correlations with the obsession interference score and time score, respectively $(r=-0.465$, $P<0.01 ; r=-0.353, P<0.05)$; and no significant correlation with the accuracy rate difference.

However, no correlation survived the FDR correction.

\section{Discussion}

We investigated the effect of stress on the neural processing associated with cognitive changes of WM in OCD and its relationship with the clinical variables. Stress impaired the WM behavioral performance in OCDs but not in HCs, specifically in more difficult WM manipulation. OCD patients showed a WM activation pattern similar to that of
HCs: increased WM-related activity in the prefrontalparietal-striatum network and decreased activity in the DMN, consistent with previous studies in HCs [22, 26-28]. The stress effect on the WM-related activity pattern in OCDs (a failure to suppress the DMN) differed from HCs (more deactivation in the $\mathrm{mPFC}$ along with less activation in the striatum). The diagnosis effect under each task condition (ME or MCE) and each stress condition (competitive or noncompetitive) on the activity was not significant, while the diagnosis and stress interaction effect on activation was significant in the right fusiform, SMA, precentral cortex, and right caudate only in the WM manipulation. OCD patients had stress-related hyperactivity in the right fusiform, SMA, precentral cortex, and right caudate, and HCs had stress-related suppression in the right caudate. Further, the clinical symptoms in OCD were associated with their task performance and the stressrelated changes of brain activation.

\section{Task Performance and Stress Effect}

OCD patients performed worse than HCs but this was only indicated by the longer reaction time in WM maintenance and manipulation and worse accuracy in WM manipulation under stress. Under stress, the task performance was 
OCD

NON-COMP
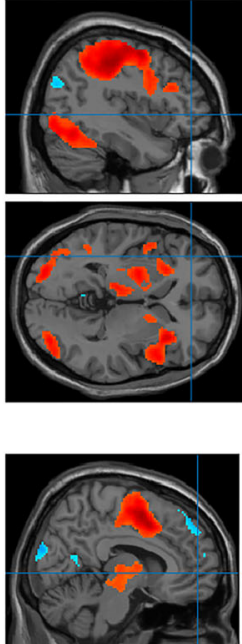

U

$\sum_{\substack{1 \\ i}}^{i} \stackrel{0}{0}$

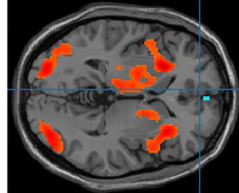

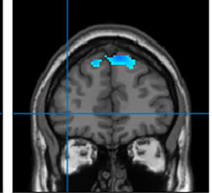
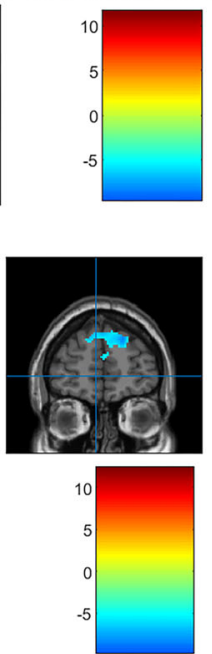

COMP
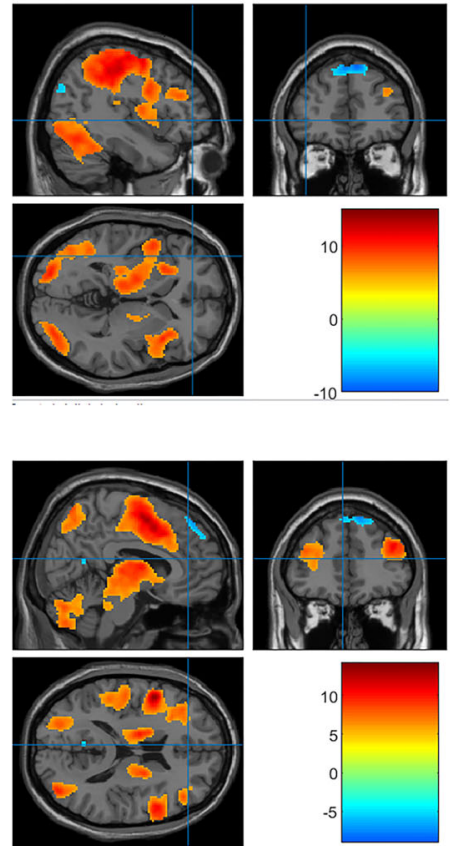

COMP vs. NON-COMP
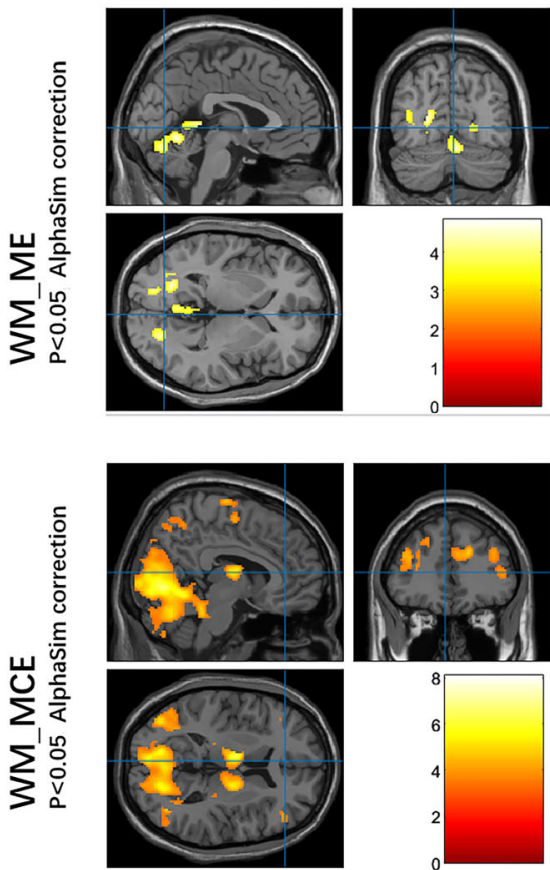

Fig. 4 Working memory-related brain activity and stress effect in the OCD group. A Activity of working memory task under competition and non-competition (left, $P<0.05$, FWE correction, cluster size $>5$ voxels). B Activity of competitive versus non-competitive conditions under different task patterns (right, $P<0.05$, AlphaSim correction for multiple comparisons). ME, maintenance evaluation; MCE, maintenance calculation evaluation; COMP, competition; NONCOMP, non-competition; FWE, whole-brain family-wise error corrected.
A
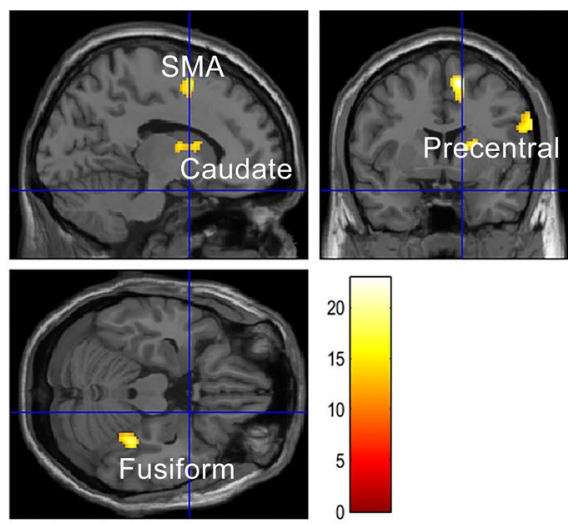

GroupxStress_Interaction effect, $P<0.05$, AlphaSim correction.
B

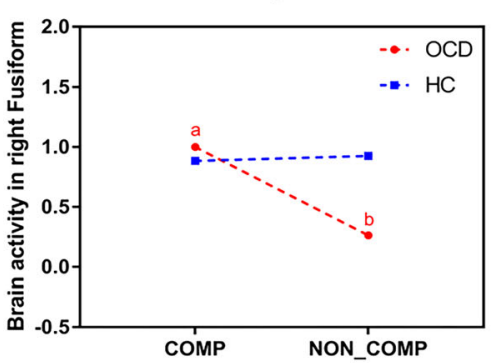

D

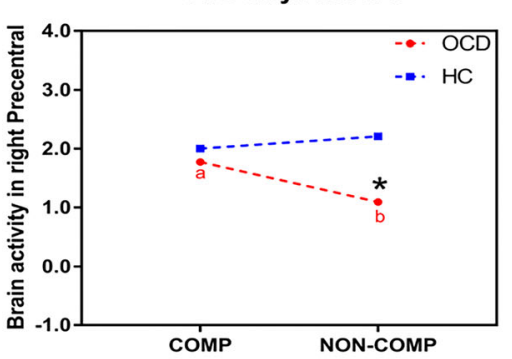

C

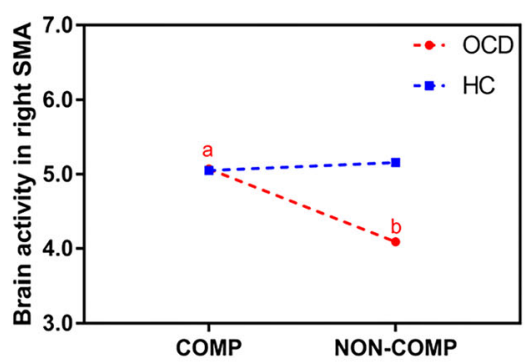

E

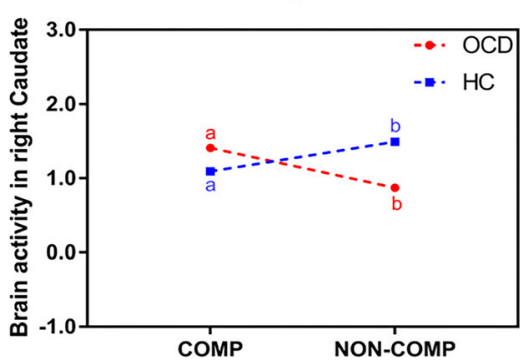

Fig. 5 Interaction effect of diagnosis and stress in brain areas. A The right fusiform, supplementary motor area, precentral, and right caudate are areas of the diagnosis and stress interaction effect. BE Activation of the right fusiform (B), supplementary motor area (C), precentral $(\mathbf{D})$, and right caudate $(\mathbf{E})$ is higher under competitive than non-competitive condition only in OCDs; $a, b$, activation of the right caudate is lower under competitive than non-competitive conditions only in HCs; *under the non-competitive condition, the BOLD signal in the right precentral is higher in HCs than OCDs. 
Table 2 The diagnosis $\times$ stress interaction effect in brain areas in the manipulation task (MCE) $(P<0.05$, AlphaSim correction).

\begin{tabular}{|c|c|c|c|c|c|c|}
\hline \multirow[t]{2}{*}{ Variables } & \multirow{2}{*}{$\begin{array}{l}\text { Cluster Size } \\
130\end{array}$} & \multirow{2}{*}{$\begin{array}{l}\text { Structure (aal) } \\
\text { Fusiform_R (aal) }\end{array}$} & \multicolumn{3}{|c|}{ Peak MNI coordinates } & \multirow{2}{*}{$\frac{\text { Peak intensity }}{4.36}$} \\
\hline & & & 32 & -36 & -20 & \\
\hline & 158 & Supp_Motor_Area_R (aal) & 8 & 4 & 56 & 4.34 \\
\hline & \multirow[t]{2}{*}{164} & Precentral_R (aal) & 62 & 4 & 28 & 3.96 \\
\hline & & Precentral_R (aal) & 54 & 4 & 24 & 3.67 \\
\hline & \multirow[t]{3}{*}{227} & Caudate_R (aal) & 18 & 18 & 2 & 3.79 \\
\hline & & Caudate_R (aal) & 14 & 10 & 12 & 3.69 \\
\hline & & Caudate_R (aal) & 14 & 0 & 12 & 3.62 \\
\hline
\end{tabular}

improved in WM maintenance and intact in the more difficult WM manipulation in HCs, while in OCDs under stress, the performance was also improved in WM maintenance but impaired in WM manipulation. Previous metaanalysis of the neuropsychology of OCD only found small negative mean effect sizes for WM across studies, which suggested that OCD patients perform worse than controls to a relatively small extent [9]. Although few previous studies have explored the role of acute stress in WM in OCD patients, several studies of HCs have shown that stress tends to increase the accuracy of WM $[29,30]$. A previous meta-analysis implied that the overall influence of acute stress on WM is deleterious, depending on stress severity, WM load, and percentage of male participants [31]. Although no significant between-group difference in the brain activity was found in WM maintenance or manipulation under stress and non-stress, the behavioral findings in the current study suggested that WM in patients with OCD was impaired to a less severe extent, consistent with the previous findings, and might be more vulnerable to stress than HCs.

\section{Brain Activation}

Less Engagement of the $\mathrm{MPFC}$ and Limbic System in HCs Under Stress

In the HCs, the stress effect on WM activations included less involvement of the mPFC (more deactivation) and the striatum (less activation) and increased activity in the cerebellum. The finding of less activation in the striatum is consistent with previous work on HCs in which stress increased activity in prefrontal and parietal cortex [29] and reduced activity in limbic areas including the ventral striatum, hypothalamus, amygdala, and hippocampus [32]. Moreover, Kogler et al. found psychosocial stress-related deactivation in the striatum and activation in the superior temporal cortex in a meta-analysis of neuroimaging studies [33]. The mPFC plays an important role in processing information about stressors and regulating the corresponding behavioral responses [34-36]. Van Leeuwen et al. investigated the responses to an emotional stimulus after inducing acute stress in healthy adults and the healthy siblings of patients with schizophrenia. They found that the HCs, but not the siblings of schizophrenics, showed reduced deactivation in the $\mathrm{mPFC}$, middle cingulate gyrus, posterior cingulate gyrus, precuneus, and superior temporal cortex following stress; these were consistent with the regions of the DMN [37]. Thus, we assumed that suppression of the mPFC might be a reassignment of neural resources to the stress response by suppressing selfreferential processes and salience detection as well [37]. However, conflicting studies have found that, after inducing stress, the dorsolateral PFC is less activated and the medial orbitofrontal cortex within the DMN is less deactivated than under the non-stress condition [38, 39]. The reason could be that an overly strong stress induced higher cortisol levels or other possible confounding factors that made the finding different from the previous [29] and our findings.

\section{Failure to Suppress the Default Mode Network in OCD Patients Under Stress}

OCD patients showed a pattern different from the HCs: regions in the DMN, including the $\mathrm{mPFC}$ and bilateral temporal cortex were more involved (less deactivation) under competitive versus non-competitive conditions. A recent study investigated the deactivated pattern of the DMN in OCDs and HCs under a transition from a resting to a non-resting context. They indicated that OCDs had a failure to suppress the DMN activity even during the emotion-provoking stimulus [40]. Using the same MIST task, Lord et al. reported less deactivation in the MPFC and orbitofrontal cortex induced by stress in postpartum OCD patients [41]. A deficit of DMN suppression has also been found in schizophrenia and depression during WM tasks [42-44], and failure of the DMN suppression is associated with a worse behavioral performance in schizophrenia and depression [42]. Thus, less suppression of the DMN under stress could be a common neural response to stress in OCD, schizophrenia, and depression and might be correlated with worse behavioral performance. 
Previous studies have generally suggested that the DMN supports an internal model for the self-referential process [45, 46]. DMN suppression plays an important role in external goal-directed cognition tasks by suppressing certain internal activity such as daydreaming and selfreferential thought [42]. A study of large-scale brain networks considered that the networks are dynamicallyshifting and resource re-allocated by neurobiological modulators to adapt to acute stress [20]. Previous functional connectivity studies of OCD reported dysconnectivity within the DMN and between networks for salience, frontoparietal and the DMN [47], and reduced connectivity within DMN subsystems [48]. Therefore, a failure of DMN suppression during cognition and under stress might be one of the neural mechanisms underlying the cognitive impairment induced by stress in OCDs.

\section{Stress-Related Hypersensitivity in OCD}

An interaction effect of diagnosis and stress was found both on behavioral performance and neural response only in WM manipulation, indicating that WM deficits in OCD are load-dependent and can be influenced by stress. Activations in the right fusiform, SMA, precentral cortex, and right caudate were significantly higher in the OCD group after inducing stress.

\section{The Fusiform Gyrus}

The lateral part of the fusiform gyrus was named as the fusiform face area (FFA) because of its specific role in face processing [49]. Few studies have investigated the function of the FFA in OCD patients. However, increased fMRI activity in the FFA has been found in schizophrenia to compensate for the damaged basic integration capability during spatial frequency-degraded face processing [50]. The FFA is hyperactivated and has greater connectivity with the amygdala in social anxiety disorder when handling the fearful face [51]. Moreover, several previous studies in healthy adults have also found interpersonal competitionrelated hyperactivation in the right fusiform and suggested its role in the social interaction process [52-54]. Thus, we assumed that the hyperactivity of the right fusiform in OCD was compensation to process the relevant information of the WM task under interpersonally competitive stress.

\section{The Supplementary Motor Area}

The right SMA showed greater activation after inducing stress in OCD patients under the manipulation task and was negatively correlated with the severity of obsession. De Vries et al. reported hyperactivity of the pre-SMA in OCD patients during a higher-level n-back task [17] and a response inhibition task [55]. They also found that right pre-SMA activation is negatively correlated with illness severity, suggesting that hyperactivity of the pre-SMA is a candidate endophenotype of OCD. The SMA, pre-SMA and the supplementary eye field compose the supplementary motor complex (SMC), which links cognition to action [56]. Studies of macaque monkeys has provided the most evidence for the anatomy and connections between the SMC and the basal ganglia (including direct, indirect, and hyper-direct pathways similar to the CSTC circuit) [56]. Lesion studies of the SMC reported difficulty in taskswitching that resulted in compulsivity [57, 58]. Thus, we assumed that the SMC takes part in the neuropathological mechanism of OCD and is activated in compensation for the stress response.

\section{The Precentral Gyrus}

The primary motor cortex is included in the precentral gyrus and is responsible for motor responses. Previous studies have found hyperactivity of the precentral gyrus in emotional regulation-related tasks in mood and anxiety disorders and high-risk individuals [59-61]. Thus, the finding of significantly lower activation of the precentral gyrus in OCDs compared with HCs under non-competitive conditions but significant hyperactivity after inducing stress in OCDs is consistent with the results of previous research and might be a compensatory mechanism for stress response.

\section{The Caudate}

Caudate dysfunction is one of the most consistent findings in OCD. Previous work on the brain morphology of OCD patients consistently found increased volume in the caudate $[62,63]$. Resting-state functional connectivity (FC) studies of OCD patients found that the decreased FC between the ventrolateral PFC and the caudate is associated with decreased cognitive flexibility [64]. A symptom provocation study reported reduced activation in the caudate nucleus [65], while activation of the caudate is considered to contribute to goal-directed behavior [66, 67]. Few previous studies have explored stress-related activation of the subcortical regions during WM in OCD patients. However, several studies have reported stress-related hyperactivity in the caudate in depressed patients, reflecting hypersensitivity to stress [44, 68]. Thus, according to the pathological model of OCD, acute stress might induce the compensatory upregulation of activity in the caudate to increase goal-directed action and to improve WM performance in OCD.

The four brain regions discussed above are associated with the pathological circuit of OCD [56, 69] and might 
also be involved in social stress processes [70]. Thus, we assumed that their activation is compensatory upregulation for the stress response. However, more research is needed to verify the existence of an OCD-specific stress network.

\section{Clinical Implications}

The accuracy rate was negatively correlated with the OCD symptom severity score, especially the accuracy rate in WM manipulation under stress, and the reaction time of all task conditions was negatively correlated with the scores on the HAMA and HAMD, which indicated that neural dysfunction related to the psychopathology of OCD is associated with the WM network dysfunction. The previous meta-analysis of resting-state fMRI showed that the frontoparietal regions are the commonly impaired circuits in both the assumed OCD model and executive and WM networks [36, 47], consistent with the behavioral findings in the current study. The contrasts of compensatory upregulation of the four areas were negatively correlated with OCD symptom severity, which indicate that patients with less severe symptoms are better able to compensate.

\section{Limitations and Future Directions}

This study has several potential limitations. First, given the limited sample size, we could not further explore the stress effect on different subtypes of OCD or gender to investigate the relationship between clinical heterogeneity and neural responses to stress. Second, more than half of the patients were taking antidepressants during fMRI scanning. Although we included medication dosage as confounding, it may still have influenced brain activity. It is necessary to recruit drug-naive patients in future. Third, the OCD and $\mathrm{HC}$ groups were well matched in demography but for age. However, we tried to remove the linear effect from all the findings and most of the participants in both groups were in their twenties. Therefore, age was less likely to be the main factor causing a between-group difference. Nevertheless, a future study with a larger sample of OCD patients and including other neuropsychiatric disorders is necessary to verify the current findings and explore their specificity over diseases. Fourth, the relationship between altered neural activity and symptoms did not conform to the expected direction, although some previous study have reported similar findings [55]. Besides, no correlation findings survived FDR correction in the current study. Therefore, we should treat all the correlation findings with caution before they are verified with further evidence.

\section{Conclusion}

The study provides evidence that OCD patients are more vulnerable to acute stress, which affects their WM-related neuro-mechanisms. The failure of suppression of the mPFC and striatum and stress-related hyperactivation of the right fusiform, SMA, precentral, and right caudate might be OCD-related psychopathological and neural responses to stress.

Acknowledgements This work was supported by the National Natural Science Foundation of China (81771443, 81370032, 81601171, and 91432304). We thank National Center for Protein Sciences at Peking University in Beijing, China, for assistance with MRI data acquisition.

Conflict of interest The authors claim that there are no conflicts of interest.

Open Access This article is licensed under a Creative Commons Attribution 4.0 International License, which permits use, sharing, adaptation, distribution and reproduction in any medium or format, as long as you give appropriate credit to the original author(s) and the source, provide a link to the Creative Commons licence, and indicate if changes were made. The images or other third party material in this article are included in the article's Creative Commons licence, unless indicated otherwise in a credit line to the material. If material is not included in the article's Creative Commons licence and your intended use is not permitted by statutory regulation or exceeds the permitted use, you will need to obtain permission directly from the copyright holder. To view a copy of this licence, visit http://creativecommons. org/licenses/by/4.0/.

\section{References}

1. Pauls DL. The genetics of obsessive-compulsive disorder: a review. Dialogues Clin Neurosci 2010, 12: 149-163.

2. Pauls DL, Abramovitch A, Rauch SL, Geller DA. Obsessivecompulsive disorder: an integrative genetic and neurobiological perspective. Nat Rev Neurosci 2014, 15: 410-424.

3. Veale D, Roberts A. Obsessive-compulsive disorder. BMJ 2014, 348: g2183.

4. Bobes J, González MP, Bascarán MT, Arango C, Sáiz PA, Bousoño M. Quality of life and disability in patients with obsessive-compulsive disorder. Eur Psychiatry 2001, 16: 239-245.

5. Brander G, Pérez-Vigil A, Larsson H, Mataix-Cols D. Systematic review of environmental risk factors for Obsessive-Compulsive Disorder: a proposed roadmap from association to causation. Neurosci Biobehav Rev 2016, 65: 36-62.

6. Kanehisa M, Kawashima C, Nakanishi M, Okamoto K, Oshita H, Masuda $\mathrm{K}$, et al. Gender differences in automatic thoughts and cortisol and alpha-amylase responses to acute psychosocial stress in patients with obsessive-compulsive personality disorder. J affect disord 2017, 217: 1-7.

7. Robbins TW, Vaghi MM, Banca P. Obsessive-Compulsive Disorder: Puzzles and Prospects. Neuron 2019, 102: 27-47.

8. Schwabe L, Wolf OT. Stress prompts habit behavior in humans. J Neurosci 2009, 29: 7191-7198. 
9. Abramovitch A, Abramowitz JS, Mittelman A. The neuropsychology of adult obsessive-compulsive disorder: a meta-analysis. Clin Psychol Rev 2013, 33: 1163-1171.

10. Dolan RJ, Dayan P. Goals and habits in the brain. Neuron 2013, 80: 312-325.

11. Liu S, Schad DJ, Kuschpel MS, Rapp MA, Heinz A. Music and Video Gaming during Breaks: Influence on Habitual versus GoalDirected Decision Making. PLoS One 2016, 11: e150165.

12. Zou S, Li CT. High-Throughput Automatic Training System for Spatial Working Memory in Free-Moving Mice. Neurosci Bull 2019, 35: 389-400.

13. Menzies L, Chamberlain SR, Laird AR, Thelen SM, Sahakian BJ, Bullmore ET. Integrating evidence from neuroimaging and neuropsychological studies of obsessive-compulsive disorder: the orbitofronto-striatal model revisited. Neurosci Biobehav Rev 2008, 32: 525-549.

14. Pauls DL, Abramovitch A, Rauch SL, Geller DA. Obsessivecompulsive disorder: an integrative genetic and neurobiological perspective. Nat Rev Neurosci 2014, 15: 410-424.

15. Saxena S, Rauch SL. Functional neuroimaging and the neuroanatomy of obsessive-compulsive disorder. Psychiatr Clin North Am 2000, 23: 563-586.

16. Banca P, Voon V, Vestergaard MD, Philipiak G, Almeida I, Pocinho $\mathrm{F}$, et al. Imbalance in habitual versus goal directed neural systems during symptom provocation in obsessive-compulsive disorder. Brain 2015, 138: 798-811.

17. de Vries FE, de Wit SJ, Cath DC, van der Werf YD, van der Borden V, van Rossum TB, et al. Compensatory frontoparietal activity during working memory: an endophenotype of obsessivecompulsive disorder. Biol Psychiatry 2014, 76: 878-887.

18. Etkin A, Wager TD. Functional neuroimaging of anxiety: a metaanalysis of emotional processing in PTSD, social anxiety disorder, and specific phobia. Am J Psychiatry 2007, 164: 1476-1488.

19. Stark EA, Parsons CE, Van Hartevelt TJ, Charquero-Ballester M, McManners H, Ehlers A, et al. Post-traumatic stress influences the brain even in the absence of symptoms: A systematic, quantitative meta-analysis of neuroimaging studies. Neurosci Biobehav Rev 2015, 56: 207-221.

20. Hermans EJ, Henckens MJAG, Joëls M, Fernández G. Dynamic adaptation of large-scale brain networks in response to acute stressors. Trends Neurosci 2014, 37: 304-314.

21. de Kloet ER, Joëls M, Holsboer F. Stress and the brain: from adaptation to disease. Nat Rev Neurosci 2005, 6: 463-475.

22. Dedovic K, Renwick R, Mahani NK, Engert V, Lupien SJ, Pruessner JC. The montreal imaging stress task: using functional imaging to investigate the effects of perceiving and processing psychosocial stress in the human brain. J Psychiatry Neurosci 2005, 30: 319-325.

23. Zhang X, Yan H, Yu H, Zhao X, Shah S, Dong Z, et al. Childhood urbanization affects prefrontal cortical responses to trait anxiety and interacts with polygenic risk for depression. bioRxiv 2019: 246876.

24. Goodman WK, Price LH, Rasmussen SA, Mazure C, Fleischmann RL, Hill CL, et al. The yale-brown obsessive compulsive scale. I. development, use, and reliability. Arch Gen Psychiatry 1989, 46: 1006-1011.

25. Hayasaka Y, Purgato M, Magni LR, Ogawa Y, Takeshima N, Cipriani A, et al. Dose equivalents of antidepressants: Evidencebased recommendations from randomized controlled trials. J Affect Disord 2015, 180: 179-184.

26. Tan HY, Chen Q, Goldberg TE, Mattay VS, Meyer-Lindenberg A, Weinberger DR, et al. Catechol-o-methyltransferase Val158Met modulation of prefrontal parietal striatal brain systems during arithmetic and temporal transformations in working memory. J Neurosci 2007, 27: 13393-13401.
27. Tan HY, Chen AG, Kolachana B, Apud JA, Mattay VS, Callicott $\mathrm{JH}$, et al. Effective connectivity of AKT1-mediated dopaminergic working memory networks and pharmacogenetics of antidopaminergic treatment. Brain 2012, 135: 1436-1445.

28. Rahm B, Kaiser J, Unterrainer JM, Simon J, Bledowski C. fMRI characterization of visual working memory recognition. Neuroimage 2014, 90: 413-422.

29. Weerda R, Muehlhan M, Wolf OT, Thiel CM. Effects of acute psychosocial stress on working memory related brain activity in men. Hum Brain Mapp 2010, 31: 1418-1429.

30. Yuen EY, Liu W, Karatsoreos IN, Feng J, McEwen BS, Yan Z. Acute stress enhances glutamatergic transmission in prefrontal cortex and facilitates working memory. Proc Natl Acad Sci U S A 2009, 106: 14075-14079.

31. Shields GS, Sazma MA, Yonelinas AP. The effects of acute stress on core executive functions: a meta-analysis and comparison with cortisol. Neurosci Biobehav Rev 2016, 68: 651-668.

32. Pruessner JC, Dedovic K, Khalili-Mahani N, Engert V, Pruessner $\mathrm{M}$, Buss C, et al. Deactivation of the limbic system during acute psychosocial stress: evidence from positron emission tomography and functional magnetic resonance imaging studies. Biol Psychiatry 2008, 63: 234-240.

33. Kogler L, Müller VI, Chang A, Eickhoff SB, Fox PT, Gur RC, et al. Psychosocial versus physiological stress - Meta-analyses on deactivations and activations of the neural correlates of stress reactions. Neuroimage 2015, 119: 235-251.

34. Amat J, Paul E, Zarza C, Watkins LR, Maier SF. Previous experience with behavioral control over stress blocks the behavioral and dorsal raphe nucleus activating effects of later uncontrollable stress: role of the ventral medial prefrontal cortex. J Neurosci 2006, 26: 13264-13272.

35. Rozeske RR, Der-Avakian A, Bland ST, Beckley JT, Watkins LR, Maier SF. The medial prefrontal cortex regulates the differential expression of morphine-conditioned place preference following a single exposure to controllable or uncontrollable stress. Neuropsychopharmacology 2009, 34: 834-843.

36. Arnsten AFT. Stress signalling pathways that impair prefrontal cortex structure and function. Nat Rev Neurosci 2009, 10: $410-422$.

37. van Leeuwen J, Vink M, Fernandez G, Hermans EJ, Joels M, Kahn RS, et al. At-risk individuals display altered brain activity following stress. Neuropsychopharmacology 2018, 43: 1954-1960.

38. Qin S, Hermans EJ, van Marle HJF, Luo J, Fernández G. Acute psychological stress reduces working memory-related activity in the dorsolateral prefrontal cortex. Biol Psychiatry 2009, 66: $25-32$.

39. Luettgau L, Schlagenhauf F, Sjoerds Z. Acute and past subjective stress influence working memory and related neural substrates. Psychoneuroendocrinology 2018, 96: 25-34.

40. Gonçalves ÓF, Soares JM, Carvalho S, Leite J, Ganho-Ávila A, Fernandes-Gonçalves A, et al. Patterns of Default Mode Network Deactivation in Obsessive Compulsive Disorder. Sci Rep 2017, 7: 44468.

41. Lord C, Steiner M, Soares CN, Carew CL, Hall GB. Stress response in postpartum women with and without obsessivecompulsive symptoms: an fMRI study. J Psychiatry Neurosci 2012, 37: 78-86.

42. Anticevic A, Cole MW, Murray JD, Corlett PR, Wang XJ, Krystal JH. The role of default network deactivation in cognition and disease. Trends Cogn Sci 2012, 16: 584-592.

43. Anticevic A, Repovs G, Barch DM. Working memory encoding and maintenance deficits in schizophrenia: neural evidence for activation and deactivation abnormalities. Schizophr Bull 2013, 39: $168-178$ 
44. Ming Q, Zhong X, Zhang X, Pu W, Dong D, Jiang Y, et al. Stateindependent and dependent neural responses to psychosocial stress in current and remitted depression. Am J Psychiatry 2017, 174: 971-979.

45. Buckner RL, Andrews-Hanna JR, Schacter DL. The brain's default network: anatomy, function, and relevance to disease. Ann N Y Acad Sci 2008, 1124: 1-38.

46. Raichle ME. The brain's default mode network. Annu Rev Neurosci 2015, 38: 433-447.

47. Gürsel DA, Avram M, Sorg C, Brandl F, Koch K. Frontoparietal areas link impairments of large-scale intrinsic brain networks with aberrant fronto-striatal interactions in OCD: a meta-analysis of resting-state functional connectivity. Neurosci Biobehav Rev 2018, 87: 151-160.

48. Beucke JC, Sepulcre J, Eldaief MC, Sebold M, Kathmann N, Kaufmann C. Default mode network subsystem alterations in obsessive-compulsive disorder. Br J Psychiatry 2014, 205: 376-382.

49. Loffler G, Yourganov G, Wilkinson F, Wilson HR. fMRI evidence for the neural representation of faces. Nat Neurosci 2005, 8: 1386-1391.

50. Silverstein SM, All SD, Kasi R, Berten S, Essex B, Lathrop KL, et al. Increased fusiform area activation in schizophrenia during processing of spatial frequency-degraded faces, as revealed by fMRI. Psychol Med 2010, 40: 1159-1169.

51. Frick A, Howner K, Fischer H, Kristiansson M, Furmark T. Altered fusiform connectivity during processing of fearful faces in social anxiety disorder. Transl Psychiatry 2013, 3: e312.

52. Assaf M, Kahn I, Pearlson GD, Johnson MR, Yeshurun Y, Calhoun VD, et al. Brain activity dissociates mentalization from motivation during an interpersonal competitive game. Brain Imaging Behav 2009, 3: 24-37.

53. Piva M, Zhang X, Noah JA, Chang SWC, Hirsch J. Distributed neural activity patterns during human-to-human competition. Front Hum Neurosci 2017, 11: 571.

54. Polosan M, Baciu M, Cousin E, Perrone M, Pichat C, Bougerol T. An fMRI study of the social competition in healthy subjects. Brain Cogn 2011, 77: 401-411.

55. de Wit SJ, de Vries FE, van der Werf YD, Cath DC, Heslenfeld DJ, Veltman EM, et al. Presupplementary motor area hyperactivity during response inhibition: a candidate endophenotype of obsessive-compulsive disorder. Am J Psychiatry 2012, 169: 1100-1108.

56. Nachev P, Kennard C, Husain M. Functional role of the supplementary and pre-supplementary motor areas. Nat Rev Neurosci 2008, 9: 856-869.

57. Husain M, Parton A, Hodgson TL, Mort D, Rees G. Self-control during response conflict by human supplementary eye field. Nat Neurosci 2003, 6: 117-118

58. Parton A, Nachev P, Hodgson TL, Mort D, Thomas D, Ordidge $\mathrm{R}$, et al. Role of the human supplementary eye field in the control of saccadic eye movements. Neuropsychologia 2007, 45: 997-1008.

59. Picó-Pérez M, Radua J, Steward T, Menchón JM, Soriano-Mas C. Emotion regulation in mood and anxiety disorders: A metaanalysis of fMRI cognitive reappraisal studies. Prog Neuropsychopharmacol Biol Psychiatry 2017, 79: 96-104.

60. Hardee JE, Cope LM, Munier EC, Welsh RC, Zucker RA, Heitzeg MM. Sex differences in the development of emotion circuitry in adolescents at risk for substance abuse: a longitudinal fMRI study. Soc Cogn Affect Neurosci 2017, 12: 965-975.

61. Saarimäki H, Gotsopoulos A, Jääskeläinen IP, Lampinen J, Vuilleumier P, Hari R, et al. Discrete neural signatures of basic emotions. Cereb Cortex 2016, 26: 2563-2573.

62. Boedhoe PS, Schmaal L, Abe Y, Ameis SH, Arnold PD, Batistuzzo MC, et al. Distinct subcortical volume alterations in pediatric and adult OCD: a worldwide meta- and mega-analysis. Am J Psychiatry 2017, 174: 60-69.

63. Tan L, Fan Q, You C, Wang J, Dong Z, Wang X, et al. Structural changes in the gray matter of unmedicated patients with obsessive-compulsive disorder: a voxel-based morphometric study. Neurosci Bull 2013, 29: 642-648.

64. Vaghi MM, Vértes PE, Kitzbichler MG, Apergis-Schoute AM, van der Flier FE, Fineberg NA, et al. Specific frontostriatal circuits for impaired cognitive flexibility and goal-directed planning in obsessive-compulsive disorder: evidence from resting-state functional connectivity. Biol Psychiatry 2017, 81: 708-717.

65. Fineberg NA, Apergis-Schoute AM, Vaghi MM, Banca P, Gillan $\mathrm{CM}$, Voon V, et al. Mapping compulsivity in the DSM-5 obsessive compulsive and related disorders: cognitive domains, neural circuitry, and treatment. Int $\mathrm{J}$ Neuropsychopharmacol 2018, 21: 42-58.

66. Jahanshahi M, Obeso I, Rothwell JC, Obeso JA. A fronto-striatosubthalamic-pallidal network for goal-directed and habitual inhibition. Nat Rev Neurosci 2015, 16: 719-732.

67. Gremel CM, Costa RM. Orbitofrontal and striatal circuits dynamically encode the shift between goal-directed and habitual actions. Nat Commun 2013, 4: 2264.

68. Admon R, Holsen LM, Aizley H, Remington A, WhitfieldGabrieli S, Goldstein JM, et al. Striatal hypersensitivity during stress in remitted individuals with recurrent depression. Biol Psychiatry 2015, 78: 67-76.

69. Schultz RT, Grelotti DJ, Klin A, Kleinman J, Van der Gaag C, Marois R, et al. The role of the fusiform face area in social cognition: implications for the pathobiology of autism. Philos Trans R Soc Lond B Biol Sci 2003, 358: 415-427.

70. Alcalá-López D, Smallwood J, Jefferies E, Van Overwalle F, Vogeley K, Mars RB, et al. Computing the social brain connectome across systems and states. Cereb Cortex 2018, 28: $2207-2232$. 